\title{
ON THE LYAPUNOV EQUATION IN BANACH SPACES AND APPLICATIONS TO CONTROL PROBLEMS
}

\author{
VU NGOC PHAT and TRAN TIN KIET
}

Received 30 August 2000 and in revised form 5 March 2001

\begin{abstract}
By extending the Lyapunov equation $A^{*} Q+Q A=-P$ to an arbitrary infinite-dimensional Banach space, we give stability conditions for a class of linear differential systems. Relationship between stabilizability and exact null-controllability is established. The result is applied to obtain new sufficient conditions for the stabilizability of a class of nonlinear control systems in Banach spaces.
\end{abstract}

2000 Mathematics Subject Classification: 93D20, 34K20, 93B05.

1. Introduction. Consider a linear system described by differential equations of the form

$$
\dot{x}(t)=A x(t), \quad t \geq 0, \quad x(0)=x_{0} \in X,
$$

where $X$ is an infinite-dimensional Banach space; $A$ is a linear operator. Over the last two decades stability problem of differential equations has been extensively studied by many researchers in qualitative theory of dynamical systems, see, for example, $[2,11,17]$ and the references therein. The classical Lyapunov theorem, which claims that the zero solution of linear system (1.1) is exponentially stable if and only if for every symmetric positive definite matrix $P$ the matrix equation

$$
A^{*} Q+Q A=-P
$$

has a symmetric positive definite matrix solution $Q$. This theorem plays an important role in the stability theory and there are several results and extensions of the Lyapunov theorem, which are closely related to the stability and Lyapunov equation (1.2), see, for example, [1, 4, 6, 14]. Moreover, the study of existence of solution of Lyapunov equation (1.2) allows us to obtain useful applications in obtaining stabilizability and controllability conditions for control systems. Among the well-known results related to these applications we mention the references $[3,7,8,9,10,13,16]$.

The purpose of this paper is twofold. Firstly, we establish equivalence between solvability of the Lyapunov equation and exponential stability of linear system (1.1) in a Banach space. Secondly, based on the Lyapunov theorem we establish a relationship between stabilizability and exact null-controllability of linear control systems and then give some applications to the exponential stabilizability of a class of nonlinear control systems in Banach spaces. The results of this paper can be considered as a further development of the results obtained earlier in $[8,10]$. 
The paper is organized as follows. In Section 2, we present the main notation, definitions and some auxiliary propositions needed later. Equivalence between solvability of the Lyapunov equation and exponential stability is given in Section 3. In Section 4, we give some applications to the exponential stabilizability of a class of nonlinear control systems in infinite-dimensional Banach spaces.

2. Preliminaries. Let $\mathbb{R}$ be the set of all real numbers, $X, U$ infinite-dimensional Banach spaces, and $X^{*}$ the topological dual space of $X$. Let $\left\langle y^{*}, x\right\rangle$ denote the value of $y^{*} \in X^{*}$ at $x \in X$. The domain, the image, the adjoint, and the inverse operator of an operator $A$ are denoted by $\mathscr{D}(A), \operatorname{Im} A, A^{*}$, and $A^{-1}$, respectively.

By $L(X, U)$ we denote the Banach space of all linear bounded operators mapping $X$ into $U$ and by $L_{2}([0, T], X)$, and $L_{2}((0, T], U)$-the Banach space of all $L_{2}$-integrable functions on $[0, T]$ taking values in $X$, and in $U$, respectively.

Let $Q \in L\left(X, X^{*}\right)$ be a duality operator. We recall that the operator $Q$ is positive definite in $X$ if $\langle Q x, x\rangle \geq 0$ for arbitrary $x \in X$, and $\langle Q x, x\rangle>0$ for $x \neq 0$. In the case if $\langle Q x, x\rangle \geq c\|x\|^{2}$ for some $c>0$ we say that $Q$ is strongly positive definite. If $X$ is a reflexive Banach space, we define the adjoint of $Q$ as the operator $Q^{*}: X \rightarrow X^{*}$. In this case, if $Q=Q^{*}$ we say that $Q$ is a selfadjoint operator. Throughout, we will denote by $\operatorname{LPD}\left(X, X^{*}\right)$ and $\operatorname{LSPD}\left(X, X^{*}\right)$ the set of all linear bounded positive definite and strongly positive definite operators mapping $X$ into $X^{*}$, respectively.

Consider linear system (1.1), where $A$ is a densely defined generator of the $C_{0^{-}}$ semigroup $S(t)$. The solution $x\left(t, x_{0}\right)$ with the initial condition $x(0)=x_{0} \in \mathscr{D}(A)$ is given by $x\left(t, x_{0}\right)=S(t) x_{0}$.

Definition 2.1. The infinitesimal generator $A$ of the $C_{0}$-semigroup $S(t)$ is exponentially stable if there exist numbers $M>0$ and $\alpha>0$, such that

$$
\|S(t)\| \leq M e^{-\alpha t}, \quad \forall t \geq 0 .
$$

DEFINITION 2.2. System (1.1) is exponentially stable if for every $x_{0} \in \mathscr{D}(A)$, there exist numbers $M>0$ and $\alpha>0$, such that

$$
\left\|S(t) x_{0}\right\| \leq M e^{-\alpha t}\left\|x_{0}\right\|, \quad \forall t \geq 0
$$

Proposition 2.3 (see [17]). Let $X$ be a Banach space, $A$ the generator of the $C_{0}$ semigroup $S(t)$. The following conditions are equivalent:

(i) system (1.1) is exponentially stable;

(ii) $A$ is exponentially stable;

(iii) for all $x_{0} \in \mathscr{D}(A): \int_{0}^{+\infty}\left\|x\left(t, x_{0}\right)\right\|^{2} d t<+\infty$.

Associated with system (1.1) we consider the following linear control system:

$$
\begin{aligned}
\dot{x} & =A x(t)+B u(t), \quad t \geq 0, \\
x(0) & =x_{0}, \quad u(t) \in U, \quad x(t) \in X,
\end{aligned}
$$

where $A$ is the generator of the $C_{0}$-semigroup $S(t)$ on some Banach space $X$ and $B \in L(U, X)$. The class of admissible controls $u$ for system (2.3) is defined by $u=$ 
$\left\{u(\cdot) \in L_{2}([0, \infty), U)\right\}$. The classical solution $x(t)$ satisfying initial condition $x(0)=$ $x_{0}$ according to some admissible control $u(\cdot) \in \mathcal{U}$ is then given by

$$
x\left(t, u, x_{0}\right)=S(t) x_{0}+\int_{0}^{t} S(t-s) B u(s) d s
$$

Definition 2.4. Control system (2.3) is exponentially stabilizable if there is an operator $K \in L(X, U)$ such that the linear system $\dot{x}(t)=(A+B K) x(t), t \geq 0$, is exponentially stable.

Definition 2.5. Control system (2.3) is exactly null-controllable in time $T>0$ if for every $x_{0} \in X$ there is an admissible control $u(t) \in U$ such that

$$
S(T) x_{0}+\int_{0}^{T} S(T-s) B u(s) d s=0
$$

In other words, if we denote by $\mathscr{C}_{T}$ the set of null-controllable points in time $T$ of system (2.3) defined by

$$
C_{T}=\left\{x_{0} \in X: S(T) x_{0}=-\int_{0}^{T} S(T-s) B u(s) d s: u(\cdot) \in u\right\}
$$

the system (2.3) is exactly null-controllable in time $T>0$ if $\mathscr{C}_{T}=X$.

In the case $A$ is the generator of an analytic semigroup $S(t)$, for $T>0$, we can define the operator $W_{T} \in L(\mathcal{U}, X)$ by

$$
W_{T}(u)=\int_{0}^{T} S^{-1}(s) B u(s) d s, \quad \forall u(\cdot) \in \mathcal{U}
$$

and we then have $C_{T}=\operatorname{Im} W_{T}$.

DEFINITION 2.6 (see [5]). A Banach space $X^{*}$ has the Radon-Nikodym property if

$$
L_{2}\left([0, T], X^{*}\right)=\left(L_{2}([0, T], X)\right)^{*} .
$$

In the sequel, we need some well-known null-controllability criteria for control system (2.3) presented in [3] for reflexive Banach spaces and then in [15] for non-reflexive Banach spaces having the Radon-Nikodym property.

Proposition 2.7 (see $[3,15]$ ). Let $X, U$ be Banach spaces, $S(t)$ the $C_{0}$-semigroup of A. Assume that $X^{*}, U^{*}$ have the Radon-Nykodym property. The following conditions are equivalent:

(i) control system (2.3) is exactly null-controllable in time $T>0$;

(ii) there exists $c>0$, for all $x^{*} \in X^{*}:\left\|W_{T}^{*} x^{*}\right\| \geq c\left\|x^{*}\right\|$;

(iii) there exists $c>0$, for all $x^{*} \in X^{*}: \int_{0}^{T}\left\|B^{*} S^{*}(s) x^{*}\right\|^{2} d s \geq c\left\|S^{*}(T) x^{*}\right\|^{2}$;

(iv) if $U$ is a Hilbert space, the operator $\int_{0}^{T} S^{-1}(s) B B^{*} S^{*^{-1}}(s) d s$ is strongly positive definite. 
3. The Lyapunov equation. Let $X$ be an arbitrary Banach space, $A$ the infinitesimal generator of the $C_{0}$-semigroup $S(t)$ and let $P \in L\left(X, X^{*}\right)$. The operator $Q \in L\left(X, X^{*}\right)$ is called a solution of the operator equation (1.2) if the following conditions hold:

$$
\langle Q A x, x\rangle+\langle Q x, A x\rangle=-\langle P x, x\rangle, \quad \forall x \in \mathscr{D}(A) .
$$

Note that if $A$ is bounded, then the above equations has the standard form

$$
A^{*} Q x+Q A x=-P x, \quad \forall x \in X,
$$

and it was shown in [4] that if $A$ is exponentially stable in a Hilbert space then the Lyapunov equation has a solution. In Theorem 3.1 below we give the equivalence between the solvability of the Lyapunov equation and the exponential stability of the linear system (1.1).

THEOREM 3.1. If for some $P \in \operatorname{LSPD}\left(X, X^{*}\right), Q \in \operatorname{LPD}\left(X, X^{*}\right)$, the Lyapunov equation holds, then the operator $A$ is exponentially stable. Conversely, if the generator $A$ is exponentially stable, then for any $P \in \operatorname{LSPD}\left(X, X^{*}\right)$, there is a solution $Q \in \operatorname{LPD}\left(X, X^{*}\right)$ of Lyapunov equation (1.2).

Proof. Assume that $Q \in \operatorname{LPD}\left(X, X^{*}\right)$ is a solution of (1.2) for some $P \in \operatorname{LSPD}\left(X, X^{*}\right)$. Let $x_{0} \in \mathscr{D}(A)$ and $x\left(t, x_{0}\right)$ be a solution of system (1.1) with the initial condition $x(0)=x_{0}$. For every $t \geq 0$, we consider the following function:

$$
V\left(x\left(t, x_{0}\right)\right)=\left\langle Q x\left(t, x_{0}\right), x\left(t, x_{0}\right)\right\rangle .
$$

We have

$$
\frac{d}{d t} V(x)=\langle Q \dot{x}, x\rangle+\langle Q x, \dot{x}\rangle=\langle Q A x, x\rangle+\langle Q x, A x\rangle=-\langle P x, x\rangle .
$$

Since $P$ is strongly positive definite, there exists a number $c>0$ such that

$$
\langle P x, x\rangle \geq c\|x\|^{2},
$$

and hence,

$$
\frac{d}{d t} V(x) \leq-c\|x\|^{2}
$$

Integrating both sides of (3.6) over $[0, t]$, we have

$$
\int_{0}^{t} \frac{d}{d s} V\left(x\left(s, x_{0}\right)\right) d s \leq-c \int_{0}^{t}\left\|x\left(s, x_{0}\right)\right\|^{2} d s,
$$

and hence

$$
V\left(x\left(t, x_{0}\right)\right)-V\left(x_{0}\right) \leq-c \int_{0}^{t}\left\|x\left(s, x_{0}\right)\right\|^{2} d s .
$$

Since $V(x) \geq 0$, we have

$$
c \int_{0}^{t}\left\|x\left(s, x_{0}\right)\right\|^{2} d s \leq V\left(x_{0}\right), \quad \forall t \geq 0 .
$$


Letting $t \rightarrow \infty$ we obtain

$$
\int_{0}^{+\infty}\left\|x\left(s, x_{0}\right)\right\|^{2} d s<+\infty
$$

which, by Proposition 2.3, implies the exponential stability of operator $A$.

Conversely, assume that the operator $A$ is exponentially stable. Take any $P \in$ $\operatorname{LSPD}\left(X, X^{*}\right)$. For each $x_{0} \in \mathscr{D}(A)$ and $t \geq 0$ we consider the operator $Q_{t} \in L\left(X, X^{*}\right)$ defined by

$$
Q_{t} x=\int_{0}^{t} S^{*}(s) P S(s) x_{0} d s
$$

We have

$$
\begin{aligned}
\left\langle Q_{t} A x_{0}, x_{0}\right\rangle+\left\langle Q_{t} x_{0}, A x_{0}\right\rangle & =\int_{0}^{t}\left[\left\langle S^{*}(s) P S(s) A x_{0}, x_{0}\right\rangle+\left\langle S^{*}(s) P S(s) x_{0}, A x_{0}\right\rangle\right] d s \\
& =\int_{0}^{t}\left[\left\langle P S(s) A x_{0}, S(s) x_{0}\right\rangle+\left\langle P S(s) x_{0}, S(s) A x_{0}\right\rangle\right] d s .
\end{aligned}
$$

Note that

$$
S(s) A=A S(s), \quad S(s) x_{0}=x\left(s, x_{0}\right),
$$

where $x\left(t, x_{0}\right)$ is the solution of system (1.1) with $x(0)=x_{0}$, we then have

$$
\begin{aligned}
\left\langle Q_{t} A x_{0}, x_{0}\right\rangle+\left\langle Q_{t} x_{0}, A x_{0}\right\rangle & =\int_{0}^{t}\left[\left\langle P A x\left(s, x_{0}\right), x\left(s, x_{0}\right)\right\rangle+\left\langle P x\left(s, x_{0}\right), A x\left(s, x_{0}\right)\right\rangle\right] d s \\
& =\int_{0}^{t} \frac{d}{d s}\langle P x(s), x(s)\rangle=\left\langle P x\left(t, x_{0}\right), x\left(t, x_{0}\right)\right\rangle-\left\langle P x_{0}, x_{0}\right\rangle .
\end{aligned}
$$

Letting $t \rightarrow+\infty$ in the above relation and noting that $x\left(t, x_{0}\right) \rightarrow 0$, we have

$$
\left\langle Q A x_{0}, x_{0}\right\rangle+\left\langle Q x_{0}, A x_{0}\right\rangle=-\left\langle P x_{0}, x_{0}\right\rangle,
$$

where the operator

$$
Q=\int_{0}^{+\infty} S^{*}(s) P S(s) d s
$$

is well defined due to the exponential stability assumption of $A$. Therefore, from the relation (3.15) it follows that $Q$ satisfies the Lyapunov equation (1.2). To complete the proof, we need to show that $Q$ is positive definite. For this, we consider

$$
\langle Q x, x\rangle=\int_{0}^{\infty}\left\langle S^{*}(s) P S(s) x, x\right\rangle d s=\int_{0}^{\infty}\langle P S(s) x, S(s) x\rangle d s .
$$

Since $P \in \operatorname{LSPD}\left(X, X^{*}\right), S(t)$ is nonsingular, we have $Q \in \operatorname{LPD}\left(X, X^{*}\right)$. The proof is complete.

REMARK 3.2. Note that if $X$ is reflexive, $P$ is selfadjoint then $Q$ is also selfadjoint. 
4. Controllability and stabilizability. Consider the linear control system (2.3), where $x(t) \in X, u(t) \in U$. Throughout this section, we assume that $X$ is a Banach space and $X^{*}$ has the Radon-Nykodym property, $U$ is a Hilbert space and the operator $A$ generates an analytic semigroup $S(t)$.

A considerable development has taken place in the problem of controllability and stabilizability of linear control system (2.3), see, for example [8, 10, 13, 16, 17, 15]. In particular, the relationship between controllability and stabilizability was presented in $[8,16]$ for systems in finite-dimensional spaces and it was shown that the exact nullcontrollability implies exponential stabilizability. It is obvious that all exactly nullcontrollable systems in finite-dimensional spaces are exponentially stabilizable, however the exponentially stabilizable system is, in general, not exactly null-controllable. For this, we need stronger notion of stabilizability in a sense of [14, 16].

DEFINITION 4.1. Linear control system (2.3), where $X, U$ are finite-dimensional, is completely stabilizable if for an arbitrary $\delta>0$ there is a matrix $K$ such that the matrix $A+B K$ is exponentially stable, that is,

$$
\left\|S_{K}(t)\right\| \leq M e^{-\delta t}, \quad \forall t \geq 0,
$$

for some $M>0$. It is well known that if a finite-dimensional linear control system is completely stabilizable in the above sense, then it is exactly null-controllable. The same definition is applied to infinite-dimensional control system (2.3) and a natural question is: to what extent does the complete stabilizability imply the exact nullcontrollability for infinite-dimensional control systems? In the infinite-dimensional control theory characterizations of controllability and stabilizability are complicated and therefore their relationships are much more complicated and require more sophisticated methods.

By a result of [8], if the linear control system (2.3), where $X, U$ are Hilbert spaces, is completely stabilizable then it is exactly null-controllable in some finite time. In the spirit of [8] using the null-controllability results, Proposition 2.7, we improve the result of [8] by the following theorem.

THEOREM 4.2. If linear control system (2.3) is completely stabilizable then it is exactly null-controllable in some finite time.

Proof. By [12, Proposition 8.3.1] we have

$$
\|S(-t)\| \leq M e^{\alpha t}, \quad t \geq 0,
$$

for some $M>0$ and $\alpha>0$. Assume that the system (2.3) is completely stabilizable, that is, for $\delta>\alpha$, there is a feedback control operator $K: X \rightarrow U$ such that the semigroup $S_{K}(t)$ generated by $(A+B K)$, satisfies the condition

$$
\left\|S_{K}(t)\right\| \leq N e^{-\delta t}, \quad t \geq 0,
$$

for some $N>0$. For every $x_{0} \in X$ and admissible control $u(t) \in \mathcal{u}$, the solution $x\left(t, x_{0}, u\right)$ of system (2.3) is given by

$$
x\left(t, x_{0}, u\right)=S(t) x_{0}+\int_{0}^{t} S(t-s) B u(s) d s,
$$


and by the feedback control $u(t)=K x(t)$ this solution is also given by

$$
x\left(t, x_{0}, u\right)=S_{K}(t) x_{0} .
$$

Therefore, we have

$$
S(t) x_{0}=S_{K}(t) x_{0}-\int_{0}^{t} S(t-s) B K S_{K}(s) d s, \quad t \geq 0 .
$$

Since the above relation holds for every $x_{0} \in X$ and using the equality (4.3), for every $x^{*} \in X^{*}$, the following estimate holds:

$$
\left\|S^{*}(t) x^{*}\right\| \leq\left\|S_{K}^{*}(t) x^{*}\right\|+\int_{0}^{t}\left\|S_{K}^{*}(s) K^{*} B^{*} S^{*}(t-s)\right\| d s,
$$

and hence

$$
\begin{aligned}
\left\|S^{*}(t) x^{*}\right\| & \leq N e^{-\delta t}\left\|x^{*}\right\|+N\left\|K^{*}\right\| \int_{0}^{t} e^{-\delta s}\left\|B^{*} S^{*}(s)\right\| d s \\
& \leq N e^{-\delta t}\left\|x^{*}\right\|+\left\|K^{*}\right\| N\left(\int_{0}^{t} e^{-2 \delta s} d s\right)^{1 / 2}\left(\int_{0}^{t}\left\|B^{*} S^{*}(s)\right\|^{2} d s\right)^{1 / 2} .
\end{aligned}
$$

Setting

$$
\beta(t)=\left(\int_{0}^{t} e^{-2 \delta s} d s\right)^{1 / 2}
$$

we see that

$$
\beta(t)=\left(\frac{1}{2 \delta}-\frac{1}{2 \delta} e^{-2 \delta t}\right)^{1 / 2}
$$

and then $\beta(t) \rightarrow(1 / \sqrt{2 \delta})$ when $t \rightarrow \infty$.

To establish the exact null-controllability of system (2.3), we assume to the contrary that the system is not null-controllable at any time $t \geq 0$. We take any $\epsilon \in(0,1)$, and set

$$
c<\left(\frac{(1-\epsilon) \sqrt{2 \delta}}{N\left\|K^{*}\right\|}\right)^{2} .
$$

Since system (2.3) is not exactly null-controllable at any time $t \geq 0$, by Proposition 2.7(iii), for that chosen number $c>0$, there is $x^{*} \in X^{*}$ such that

$$
\int_{0}^{t}\left\|B^{*} S(s) x^{*}\right\| d s<c\left\|S^{*}(t) x^{*}\right\|^{2} .
$$

From the above inequality, it follows that $x^{*} \neq 0$ and we can consider $\left\|x^{*}\right\|=1$. On the other hand, in view of (4.8), we have the following estimate:

$$
\left\|S^{*}(t) x^{*}\right\|<N e^{-\delta t}+\sqrt{c} N \beta(t)\left\|K^{*}\right\|\left\|S^{*}(t) x^{*}\right\|,
$$

or equivalently

$$
1<\frac{N e^{-\delta t}}{\left\|S^{*}(t) x^{*}\right\|}+\sqrt{c} N\left\|K^{*}\right\| \beta(t) .
$$


Since

$$
1=\left\|S^{*}(-t) S^{*}(t) x^{*}\right\| \leq\left\|S^{*}(-t)\right\|\left\|S^{*}(t) x^{*}\right\|
$$

and using (4.2), we have

$$
\frac{1}{\left\|S^{*}(t) x^{*}\right\|} \leq\left\|S^{*}(-t)\right\| \leq M e^{\alpha t} .
$$

Combining (4.11), (4.14), and (4.16) gives

$$
1-\sqrt{c} N \beta(t)\left\|K^{*}\right\|<N M e^{-(\delta-\alpha) t}, \quad \forall t \geq 0 .
$$

Letting $t \rightarrow+\infty$, and noting $\delta>\alpha$, the right-hand side goes to zero and we then have

$$
\epsilon \leq 1-\sqrt{c} N \frac{1}{\sqrt{2 \delta}}\left\|K^{*}\right\|<0 .
$$

The last inequality contradicts the choice of numbers $\epsilon, c$ by (4.11). The system is exactly null-controllable.

In the sequel, we prove that if linear control system (2.3) is null-controllable then it is exponentially stabilizable by some linear feedback control $K: X \rightarrow U$.

THEOREM 4.3. If control system (2.3) is exactly null-controllable in some finite time, then the system is exponentially stabilizable.

Proof. Assume that the system is exactly null-controllable in $T>0$. The operator $Q \in L\left(X^{*}, X\right)$ given by

$$
Q x^{*}=\int_{0}^{T} S^{-1}(s) B B^{*} S^{*^{-1}}(s) x^{*} d s,
$$

is, by Proposition 2.7(iv), well defined and strictly positive definite. Therefore, the inverse operator $Q^{-1}: X \rightarrow X^{*}$ is also well defined. We will prove that the control system (2.3) is exponentially stabilizable by the feedback control

$$
u(t)=K x(t)=-B^{*} Q^{-1} x(t) .
$$

It is enough to show that the operator $Q$ satisfies the Lyapunov equation (1.2) in the dual space $X^{*}$ with $\mathscr{A}=(A+B K)^{*}$ for some $P \in \operatorname{LSPD}\left(X^{*}, X\right)$ and by Theorem $3.1, \mathscr{A}$ and then $(\mathscr{A})^{*}=A+B K$ is exponentially stable. For this, we have to prove that

$$
\mathscr{A}^{*} Q x^{*}+Q \mathscr{A} x^{*}=-P x^{*}, \quad \forall x^{*} \in X^{*} .
$$

Indeed, we consider

$$
Q A^{*}+A Q=A \int_{0}^{T} S^{-1}(s) B B^{*} S^{*^{-1}}(s) d s+\int_{0}^{T} S^{-1}(s) B B^{*} S^{*^{-1}}(s) A^{*} d s .
$$

Since

$$
\frac{d}{d t} S^{-1}(t)=-A S^{-1}(t), \quad \frac{d}{d t} S^{*^{-1}}(t)=-S^{*^{-1}}(t) A^{*},
$$


we have

$$
Q A^{*}+A Q=-\int_{0}^{T} \frac{d}{d s}\left[S^{-1}(s) B B^{*} S^{*^{-1}}(s)\right] d s=B B^{*}-S^{-1}(T) B B^{*} S^{*^{-1}}(T) .
$$

Consider the relation $Q \mathscr{A}+\mathscr{A}^{*} Q$. Since $Q$ is a selfadjoint operator, we see that

$$
Q \mathscr{A}+\mathscr{A}^{*} Q=Q\left(A-B B^{*} Q^{-1}\right)^{*}+\left(A-B B^{*} Q^{-1}\right) Q=Q A^{*}+A Q-2 B B^{*} .
$$

From (4.24), it follows that

$$
Q \mathscr{A}+\mathscr{A}^{*} Q=-P
$$

where

$$
P:=\left[B B^{*}+S^{-1}(T) B B^{*} S^{*^{-1}}(T)\right] .
$$

Therefore, for every $x^{*} \in X^{*}$, we have

$$
\mathscr{A}^{*} Q x^{*}+Q \mathscr{A} x^{*}=-P x^{*}
$$

as desired. To complete the proof, we show that $P$ is strictly positive definite. This follows from the following relations

$$
\begin{aligned}
\left\langle P x^{*}, x^{*}\right\rangle & =\left\langle B B^{*} x^{*}, x^{*}\right\rangle+\left\langle S^{-1}(T) B B^{*} S^{*^{-1}}(T) x^{*}, x^{*}\right\rangle \\
& =\left\|B^{*} x^{*}\right\|^{2}+\left\|B^{*} S^{*^{-1}}(T) x^{*}\right\|^{2}, \quad \forall x^{*} \in X^{*}
\end{aligned}
$$

Since

$$
\left(W_{T}^{*} x^{*}\right)(s)=B^{*} S^{*-1}(s) x^{*}, \quad \forall s \in[0, T],
$$

and using Proposition 2.7(ii), we have

$$
\left\langle P x^{*}, x^{*}\right\rangle \geq\left\|B^{*} S^{*^{-1}}(T) x^{*}\right\|^{2}=\left\|\left(W_{T}^{*} x^{*}\right)(T)\right\|^{2} \geq c_{1}\left\|x^{*}\right\|^{2},
$$

for some positive number $c_{1}>0$. The proof is complete.

REMARK 4.4. It is worth noting that Theorem 4.2 was presented in [17] for the case $X$ is a Hilbert space and the proof therein is based on the linear regulator optimization problem so that it is quite different from ours.

EXAMPLE 4.5. Consider a control system of the form

$$
\begin{aligned}
& \dot{x}(t)=A x(t)+B u(t), \quad t \in \mathbb{R}^{+}, \\
& x(t) \in X=l_{2}, \quad u(t) \in U=l_{2},
\end{aligned}
$$

where $l_{2}$ is the space of all sequences $\beta=\beta_{1}, \beta_{2}, \ldots$, with the norm

$$
\|\beta\|=\left(\sum_{i=1}^{\infty}\left|\beta_{i}\right|^{2}\right)^{1 / 2}<+\infty
$$

and the operators $A, B$ are given by

$$
\begin{aligned}
& A:\left(\beta_{1}, \beta_{2}, \ldots\right) \in l_{2} \longrightarrow\left(\beta_{2}, \ldots\right) \in l_{2}, \\
& B:\left(\beta_{1}, \beta_{2}, \ldots\right) \in l_{2} \longrightarrow(0,0, \underbrace{\ldots}_{N}, \beta_{1}, \beta_{2}, \ldots) \in l_{2}, \quad N>0 .
\end{aligned}
$$


Since $A^{N} B U=l_{2}$, the system is exactly null-controllable and hence the system is stabilizable.

5. Stabilizability of nonlinear control systems. As an application, we study stabilizability problem of a nonlinear control system of the form

$$
\dot{x}=A x+B u+f(x, u), \quad t \geq 0 .
$$

In this section, we also assume that $X$ is a Banach space and $X^{*}$ has the RadonNykodym property, $U$ is a Hilbert space, $f(x, u): X \times U \rightarrow X$ is some given nonlinear function satisfying the following comparable condition

$$
\|f(x, u)\| \leq a\|x\|+b\|u\|, \quad \forall(x, u) \in(X \times U),
$$

for some $a>0, b>0$. We recall that control system (5.1) is stabilizable by a feedback control $u(t)=K x(t), K \in L(X, U)$ if the uncontrolled system $\dot{x}(t)=(A x+K B) x+$ $f(x, K x)$ is asymptotically stable in Lyapunov sense.

The following theorem gives a sufficient condition for stabilizability of nonlinear control system (5.1) in the case $A$ is a stable operator.

THEOREM 5.1. Assume that $A$ is exponentially stable and the condition (5.2) is satisfied. Let $P, Q \in \operatorname{LPD}\left(X, X^{*}\right)$ be the operators satisfying the Lyapunov equation (1.2), where $Q=Q^{*}$ and $\langle P x, x\rangle \geq \alpha\|x\|^{2}$, for all $x \in X, \alpha>0$. The nonlinear control system (5.1) is stabilizable by the feedback control $u(t)=-\beta B^{*} Q x(t)$ if

$$
0<\beta<\frac{\alpha-2 a\|Q\|}{2 b\|B\|\|Q\|^{2}} .
$$

Proof. Let $x\left(t, x_{0}\right)$ be any solution of system (5.1). Let $Q \in \operatorname{LPD}\left(X, X^{*}\right)$ be a solution of the Lyapunov equation (1.2). We consider the following function of the form

$$
V\left(x\left(t, x_{0}\right)\right)=\left\langle Q x\left(t, x_{0}\right), x\left(t, x_{0}\right)\right\rangle .
$$

and we prove that this function is a Lyapunov function for the system (5.1). Indeed, we have

$$
\begin{aligned}
\frac{d}{d t} V(x)= & \langle Q \dot{x}, x\rangle+\langle Q x, \dot{x}\rangle \\
= & \left\langle Q\left(A x-\beta B B^{*} Q x+f(x, u)\right), x\right\rangle+\left\langle Q x, A x-\beta B B^{*} Q+f(x, u)\right\rangle \\
= & -\langle P x, x\rangle-\beta\left\langle Q B B^{*} Q x, x\right\rangle-\beta\left\langle Q x, B B^{*} Q x\right\rangle \\
& +\langle Q f(x, u), x\rangle+\langle Q x, f(x, u)\rangle .
\end{aligned}
$$

Since $Q$ is selfadjoint, by conditions (5.2) and (5.3), we obtain the following estimate

$$
\begin{aligned}
\frac{d}{d t} V(x) & \leq-\alpha\|x\|^{2}-2 \beta\left\|B^{*} Q x\right\|^{2}+2\|Q\|\left(a\|x\|+b \beta\left\|B^{*}\right\|\|Q\|\|x\|\right)\|x\| \\
& \leq-\left(\alpha-2 b \beta\|Q\|^{2}\|B\|-2 a\|Q\|\right)\|x\|^{2} \leq-\delta\|x\|^{2}
\end{aligned}
$$

where

$$
\delta=\alpha-2 b \beta\|Q\|^{2}\|B\|-2 a\|Q\|>0,
$$

as desired. 
In Theorem 5.2, we give another sufficient condition for the stabilizability of system (5.1) in the case $A$ is not stable, but the associated linear control system (2.3) is exactly null-controllable and the nonlinear perturbation $f(\cdot)$ is small enough.

THEOREM 5.2. Assume that the linear control system (2.3) is exactly null-controllable. The system (5.1) is stabilizable for some appropriate numbers $a>0, b>0$ satisfying the condition (5.2).

Proof. By Theorem 4.3, the linear control system (2.3) is stabilizable and then there is an operator $D \in L(X, U)$ such that the operator $\mathscr{A}=A+B D$ is exponentially stable. Let $P, Q \in \operatorname{LPD}\left(X, X^{*}\right)$ be a solution pair of the Lyapunov equation with respect to $A$, where $\langle P x, x\rangle \leq \alpha\|x\|^{2}, Q=Q^{*}$. Consider the Lyapunov function $V(t, x)=$ $\langle Q x, x\rangle$, for the nonlinear control system (5.1). By the same arguments used in the proof of Theorem 5.1, we have

$$
\begin{aligned}
\frac{d}{d t} V(t, x) & \leq-\alpha\|x\|^{2}+2\langle Q f(x, D x), x\rangle \\
& \leq-[\alpha-2(a\|Q\|+b\|D\|)]\|x\|^{2}=-\delta\|x\|^{2},
\end{aligned}
$$

where $\delta=\alpha-2(a\|Q\|+b\|D\|)$. We now choose $a, b>0$ such that $\delta>0$, that is,

$$
a\|Q\|+b\|D\|<\frac{\alpha}{2} .
$$

Then the nonlinear system (5.1) is stabilizable. The proof is complete.

6. Conclusions. In this paper an extension of the Lyapunov equation in Banach spaces was studied. A relationship between stabilizability and exact null-controllability of linear systems in Banach spaces was established. Some applications to stabilizability problem of a class of nonlinear control systems in infinite-dimensional Banach spaces were also given.

\section{REFERENCES}

[1] W. Arendt, F. Räbiger, and A. Sourour, Spectral properties of the operator equation $A X+$ $X B=Y$, Quart. J. Math. Oxford Ser. (2) 45 (1994), no. 178, 133-149.

[2] T. A. Burton and G. Makay, Marachkov type stability results for functional-differential equations, Electron. J. Qual. Theory Differ. Equ. (1998), 1-17.

[3] R. F. Curtain and A. J. Pritchard, Infinite Dimensional Linear Systems Theory, Lecture Notes in Control and Information Sciences, vol. 8, Springer-Verlag, Berlin, 1978.

[4] R. Datko, Extending a theorem of A. M. Liapunov to Hilbert spaces, J. Math. Anal. Appl. 32 (1970), 610-616.

[5] J. Diestel and J. J. Uhl Jr., Vector Measures, Mathematical Surveys, no. 15, American Mathematical Society, Rhode Island, 1977.

[6] J. A. Goldstein, On the operator equation $A X+X B=Q$, Proc. Amer. Math. Soc. 70 (1978), no. 1, 31-34.

[7] T. T. Kiet and V. N. Phat, On the stabilizability of nonlinear systems in infinite-dimensional spaces, Nonl. Funct. Anal. Appl. 4 (1999), 73-85.

[8] G. Megan, On the Stabilizability and Controllability of Linear Dissipative Systems in Hilbert Spaces, vol. 32, S.E.F., Universitate din Timisoara, 1975.

[9] S. Nikitin, Global Controllability and Stabilization of Nonlinear Systems, Series on Advances in Mathematics for Applied Sciences, vol. 20, World Scientific Publishing, New Jersey, 1994. 
[10] V. N. Phat, On the stability of time-varying differential equations, Optimization 45 (1999), no. 1-4, 237-254.

[11] A. J. Pritchard and J. Zabczyk, Stability and stabilizability of infinite-dimensional systems, SIAM Rev. 23 (1981), no. 1, 25-52.

[12] S. Rolewicz, Functional Analysis and Control Theory. Linear Systems, Mathematics and Its Applications (East European Series), vol. 29, D. Reidel Publishing, Dordrecht, 1987, translated from Polish by Ewa Bednarczuk.

[13] D. L. Russel, Controllability and stabilizability theory for PDEs: recent-progress and open questions, SIAM Rev. 20 (1978), 639-739.

[14] W. M. Wonham, On pole assignment in multi-input controllable linear systems, IEEE Trans. Automat. Control 12 (1967), 660-665.

[15] H. Xuejiao and C. Zhenchao, Controllability of linear systems in non-reflexive Banach spaces, Northeast Math. J. 15 (1999), 459-464.

[16] J. Zabczyk, Complete stabilizability implies exact controllability, Seminaru Ecuati Functionale 38 (1977), 1-10.

[17] _ Mathematical Control Theory: An Introduction, Birkhäuser Boston, Massachusetts, 1992.

Vu NGoc Phat: Institute of Mathematics, Box 631, Bo Ho, 10.000, Hanoi, Vietnam

E-mail address: vnphat@hanimath.ac.vn

Tran Tin Kiet: Department of Mathematics, Quinhon Pedagogical University, Qui NHON CITY, BINH DINH, VIETNAM 


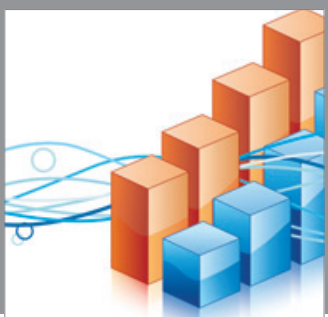

Advances in

Operations Research

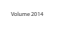

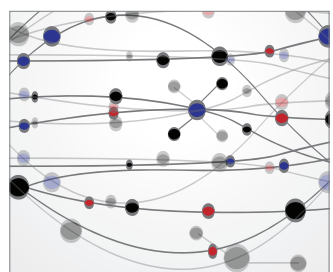

\section{The Scientific} World Journal
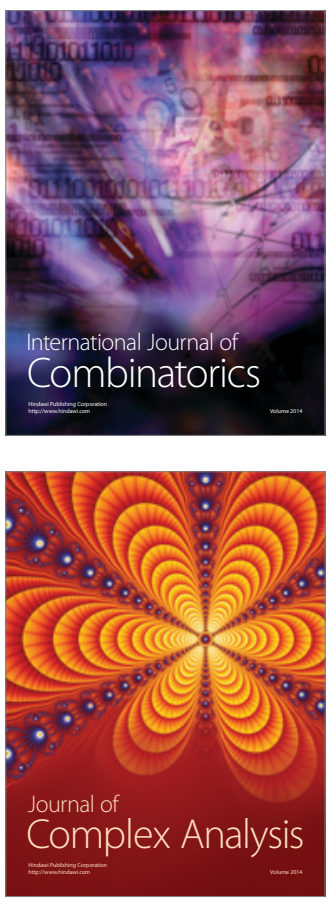

International Journal of

Mathematics and

Mathematical

Sciences
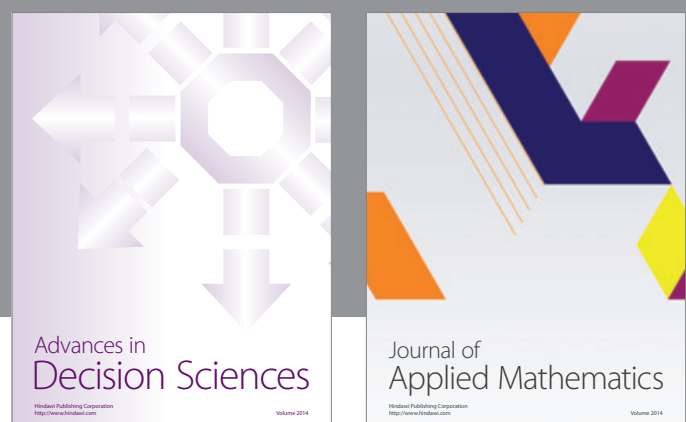

Journal of

Applied Mathematics
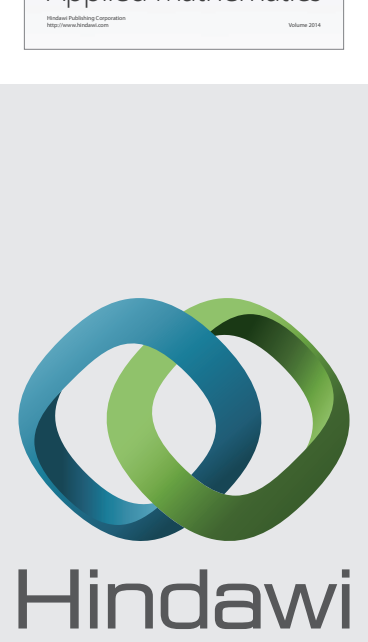

Submit your manuscripts at http://www.hindawi.com
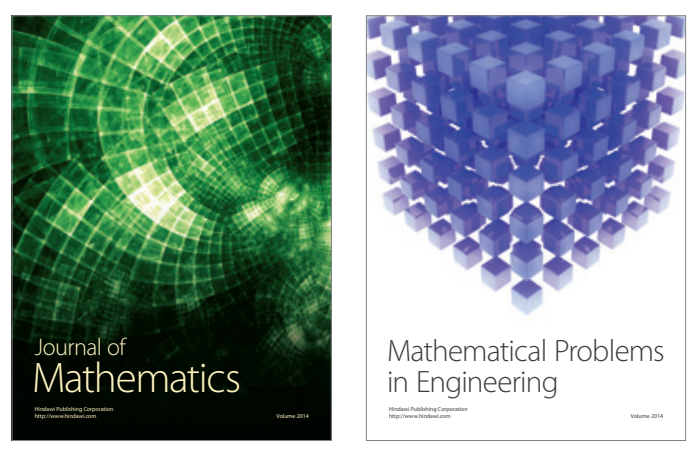

Mathematical Problems in Engineering
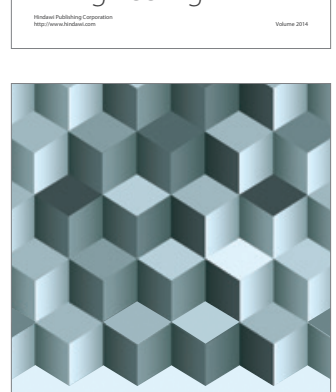

Journal of

Function Spaces
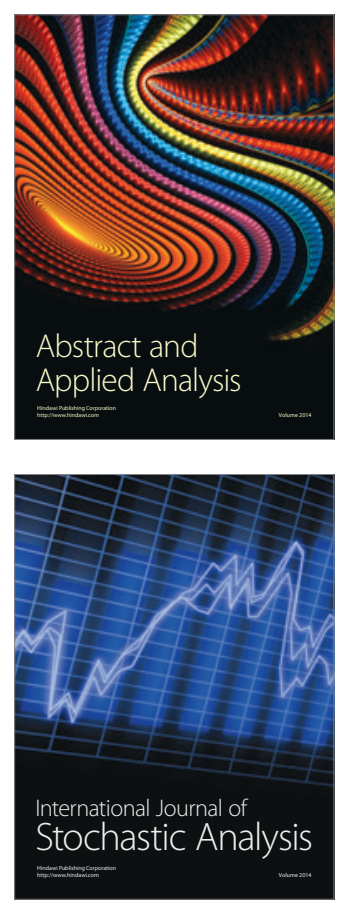

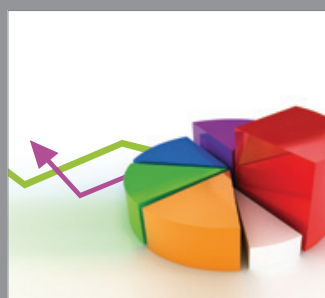

ournal of

Probability and Statistics

Promensencen
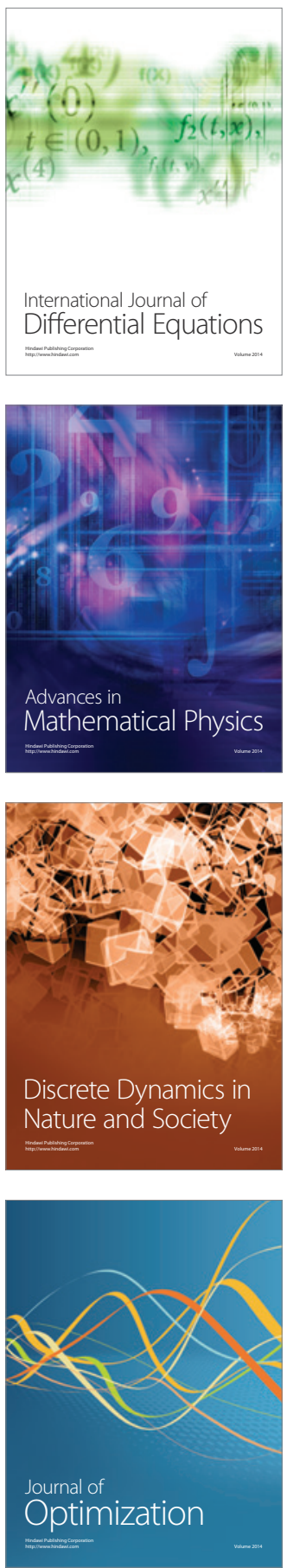